\title{
The use of check dams for soil restoration at watershed level: a century of history and perspectives
}

\author{
Manuel Esteban Lucas-Borja (1), Guillaume Piton (2), Mary Nichols (3), Carlos Castillo (4), Demetrio Zema (5), \\ and Yang Yu (6) \\ (1) Castilla La Mancha University, Spain, (2) Univ. Grenoble Alpes, IRSTEA, France, (3) USDA-ARS, Southwest Watershed \\ Research Centre, USA, (4) University of Córdoba, Spain, (5) Mediterranea University of Reggio Calabria, Italy, (6) Institute \\ of Water Resources and Hydropower Research, China
}

Check dams are transverse structures designed and built in watersheds mainly to control water and sediment flows, conserve soil and improve land. Their stabilization role across stream-beds and gullies have been well known since many years. National, regional and local governments have spent in the last century, and still currently spend, important funds for maintenance and new implementations of check dams as basin scale erosion-control measures throughout the world. However, some projects experience disappointing results due to many different circumstances, such as poor construction quality, inadequate check dam location and lack of adequate design criteria. In addition, these structures induce secondary effects: for instance, different studies have pointed that check dams represent one of the most dominant forms of human impact upon mountain fluvial systems, as they disrupt the downstream transfer of water and sediments; observations of channel cross sections and bed material in several studies for instance indicate that check dams may increase erosion downstream. Furthermore, in spite of many and eminent studies focusing on laboratory and field researches, the complex hydraulic functioning of the structures (in particular for open check dams, proposed to smooth the adverse effects of the traditional structures) is not completely understood. Thus, there is a lack of full knowledge to optimize existing dams and define the best-adapted design to a given site, also considering the variety of factors (materials, size, number, type, etc.) of these engineering works and effects (morphological, hydraulic, sedimentary, ecological and so on) played by them.

In this communication, we present the preliminary results of an international review process considering more than 200 papers to highlight problems and benefits of check dam construction all over the world. We aim to achieve a detailed comprehension of check dams' effects at watershed scale in soil restoration schemes from the analysis of results reported in global literature (conceptual thinking, field observations, laboratory simulations), in order to contribute to filling the knowledge gaps identified above. This work is intended to represent a starting point to a future perspective to increase the confidence in developing check dams as restoration tools at watershed scale. 